PROCEEDINGS OF THE

AMERICAN MATHEMATICAL SOCIETY

Volume 130, Number 11, Pages 3255-3258

S 0002-9939(02)06626-1

Article electronically published on April 22, 2002

\title{
LOCAL DUAL SPACES OF BANACH SPACES OF VECTOR-VALUED FUNCTIONS
}

\author{
MANUEL GONZÁLEZ AND ANTONIO MARTÍNEZ-ABEJÓN
}

(Communicated by Jonathan M. Borwein)

\begin{abstract}
We show that $L_{\infty}\left(\mu, X^{*}\right)$ is a local dual of $L_{1}(\mu, X)$, and $L_{1}\left(\mu, X^{*}\right)$ is a local dual of $L_{\infty}(\mu, X)$, where $X$ is a Banach space. A local dual space of a Banach space $Y$ is a subspace $Z$ of $Y^{*}$ so that we have a local representation of $Y^{*}$ in $Z$ satisfying the properties of the representation of $X^{* *}$ in $X$ provided by the principle of local reflexivity.
\end{abstract}

\section{INTRODUCTION}

The principle of local reflexivity shows that there is a close relation between a Banach space $X$ and its second dual $X^{* *}$ from a finite dimensional point of view. This means that $X$ can be considered "locally" as a dual of $X^{*}$.

In [6] we introduced the local dual spaces of $X$. These subspaces satisfy the thesis of the principle of local reflexivity in full force: $X^{*}$ is finitely dual representable in any of its local dual spaces by means of $\varepsilon$-isometries that fix points. Moreover, in [5] we studied the polar property for subspaces $Z$ of $X^{*}$ as a test to check if $X^{*}$ is finitely dual representable in $Z$. We observe that being a local dual is strictly stronger than satisfying the polar property.

It was proved in 66 that $\ell_{1}\left(X^{*}\right)$ is a local dual of $\ell_{\infty}(X)$, and $\ell_{\infty}(X)$ is a local dual of $\ell_{1}\left(X^{*}\right)$. Moreover, assuming the continuum hypothesis $2^{\omega}=\omega_{1}, C[0,1]$ is a local dual of $L_{1}[0,1]$ and $L_{1}[0,1]$ is a local dual of $C[0,1]$. Also every separable space $X$ with the metric approximation property has a local dual with the metric approximation property.

Here we describe local dual spaces for some spaces of vector-valued functions. Let $\mu$ be a finite measure. We show that $L_{1}\left(\mu, X^{*}\right)$ is a local dual of $L_{\infty}(\mu, X)$, and $L_{\infty}\left(\mu, X^{*}\right)$ is a local dual of $L_{1}(\mu, X)$. Note that $L_{1}(\mu, X)^{*}$ can be described as a space of $X^{*}$-valued, weak ${ }^{*}$-measurable functions [2], and $L_{\infty}(\mu, X)^{*}$ can be described as a direct sum of $L_{1}\left(\mu, X^{*}\right)$ and a certain subspace of singular elements 11. But these descriptions are not always manageable, especially in the latter case. Previously, Díaz [3] studied the duality between $L_{\infty}(\mu, X)$ and $L_{1}\left(\mu, X^{*}\right)$, but our results are stronger and our proofs are more natural.

Received by the editors June 5, 2001.

2000 Mathematics Subject Classification. Primary 46B10, 46B20; Secondary 46B04, 46B08.

Key words and phrases. Local dual space, local reflexivity, norming subspace, Banach spaces of vector-valued functions.

This work was supported in part by DGICYT Grant PB 97-0349. 
In the paper $X$ and $Y$ are Banach spaces, $B_{X}$ the closed unit ball of $X, S_{X}$ the unit sphere of $X$, and $X^{*}$ the dual of $X$. We identify $X$ with a subspace of $X^{* *}$. By a subspace we always mean a closed subspace. We denote by $\mathcal{B}(X, Y)$ the space of all (bounded linear) operators from $X$ into $Y$. Given $T \in \mathcal{B}(X, Y), R(T)$ is the range of $T$, and $T^{*}$ is the conjugate operator of $T$.

Given a number $0<\varepsilon<1$, an operator $T \in \mathcal{B}(X, Y)$ is an $\varepsilon$-isometry if it satisfies $(1+\varepsilon)^{-1}<\|T x\|<1+\varepsilon$ for all $x \in S_{X}$. A space $X$ is said to be finitely representable in $Y$ if for each $\varepsilon>0$ and each finite dimensional subspace $M$ of $X$ there is an $\varepsilon$-isometry $T: M \longrightarrow Y$.

\section{MAIN RESUlts}

Local dual spaces of Banach spaces were introduced in [6 Definition 2.1] as follows:

Definition 1. A subspace $Z$ of $X^{*}$ is said to be a local dual space of $X$ if for every couple of finite dimensional subspaces $F$ of $X^{*}$ and $G$ of $X$, and every number $0<\varepsilon<1$, there is an $\varepsilon$-isometry $L: F \longrightarrow Z$ satisfying the following conditions:

(a) $\langle L f, x\rangle=\langle f, x\rangle$ for all $x \in G$ and all $f \in F$, and

(b) $L(f)=f$ for all $f \in F \cap Z$.

Obviously, $X^{*}$ is a local dual of $X$. Moreover, the principle of local reflexivity establishes that every isometric predual is a local dual of $X^{*}$, and the principle of local reflexivity for ultrapowers [7, Theorem 7.3] establishes that $\left(X^{*}\right)_{\mathfrak{U}}$ is a local dual of $X_{\mathfrak{U}}$. Observe that we do not have a description of $\left(X_{\mathfrak{U}}\right)^{*}$.

Given a couple of subspaces $Z$ of $X^{*}$ and $G$ of $Z^{*}$, an operator $L: G \longrightarrow X^{* *}$ is said to be an extension operator if $\left.L f\right|_{Z}=f$, for every $f \in G$. The following characterization of the local dual spaces of a Banach space will be the key to proving our results.

Theorem 2 ([6, Theorem 2.5]). A subspace $Z$ of $X^{*}$ is a local dual of $X$ if (and only if) for every couple of finite dimensional subspaces $F$ of $X^{*}$ and $G$ of $X$, and every $0<\varepsilon<1$, there is an $\varepsilon$-isometry $L: F \longrightarrow Z$ such that

(a) $|\langle L f, x\rangle-\langle f, x\rangle|<\varepsilon\|f\|\|x\|$, for all $x \in G$ and all $f \in F$, and

(b) $\|L(f)-f\| \leq \varepsilon\|f\|$ for all $f \in F \cap Z$.

The relation "being a local dual" is symmetric. Let $\hat{x}$ denote the vector $x \in X$ as an element of $X^{* *}$. We define a map $\Upsilon: X \longrightarrow Z^{*}$ by $\Upsilon(x)=\left.\hat{x}\right|_{Z}$.

Proposition 3 ([6, Proposition 2.10(b)]). Let $Z$ be a local dual of $X$. Then $\Upsilon(X)$ is a local dual of $Z$ which is isometric to $X$.

Here is our main result.

Theorem 4. Let $\mu$ be a finite measure. Then:

(a) $L_{1}\left(\mu, X^{*}\right)$ is a local dual of $L_{\infty}(\mu, X)$.

(b) $L_{\infty}\left(\mu, X^{*}\right)$ is a local dual of $L_{1}(\mu, X)$.

Proof. The set $\mathcal{S}_{1}\left(\mu, X^{*}\right)$ of all functions $g=\sum_{n=1}^{\infty} \chi_{A_{n}} \otimes x_{n}^{*}$, where $\left(A_{n}\right)$ is a disjoint sequence of measurable sets and $\left(x_{n}^{*}\right)$ is a sequence in $X^{*}$ so that $\sum_{n} \mu\left(A_{n}\right)\left\|x_{n}^{*}\right\|<\infty$, is dense in $L_{1}\left(\mu, X^{*}\right)$. Analogously, since each $f \in L_{\infty}(\mu, X)$ is essentially valued in a separable subspace of $X$, the set $\mathcal{S}_{\infty}(\mu, X)$ of all functions 
$f=\sum_{n=1}^{\infty} \chi_{A_{n}} \otimes x_{n}$, where $\left(A_{n}\right)$ is a disjoint sequence of measurable sets and $\left(x_{n}\right)$ is a bounded sequence in $X$, is dense in $L_{\infty}(\mu, X)$.

(a) In order to apply Theorem 2, we fix $0<\varepsilon<1$ and finite dimensional subspaces $F$ of $L_{\infty}(\mu, X)^{*}$ and $G$ of $L_{\infty}(\mu, X)$. Without loss of generality we can assume that $G$ is large enough so that, for every $\phi \in F$,

$$
\|\phi\| \leq(1+\varepsilon) \sup \left\{|\langle\phi, f\rangle|: f \in S_{G}\right\} .
$$

Let $\left\{\phi_{1}, \ldots, \phi_{l}\right\}$ be a basis for $F \cap L_{1}\left(\mu, X^{*}\right)$. We take $\left\{\phi_{1}^{*}, \ldots, \phi_{l}^{*}\right\}$ in $L_{\infty}(\mu, X)^{* *}$ so that $\left\langle\phi_{i}^{*}, \phi_{j}\right\rangle=\delta_{i j}$, for $i, j=1, \ldots, l$. Let $M=\sup \left\|\phi_{i}^{*}\right\|$. We select $f_{1}, \ldots, f_{l}$ in $\mathcal{S}_{1}\left(\mu, X^{*}\right)$ so that $\left\|\phi_{j}-f_{j}\right\|<\varepsilon /(2 l M)$, for $j=1, \ldots, l$.

We define an operator $T$ on $L_{\infty}(\mu, X)^{*}$ by

$$
T(\phi)=\phi-\sum_{j=1}^{l}\left\langle\phi_{j}^{*}, \phi\right\rangle\left(\phi_{j}-f_{j}\right) .
$$

Then $\|I-T\|<\varepsilon / 2$ and $T\left(\phi_{j}\right)=f_{j}$, for $j=1, \ldots, l$.

Analogously, let $\left\{h_{1}, \ldots, h_{k}\right\}$ be a basis for $G$. We can select $g_{1}, \ldots, g_{k}$ in $\mathcal{S}_{\infty}(\mu, X)$, and define an operator $S$ on $L_{\infty}(\mu, X)$ such that $\|I-S\|<\varepsilon / 2$ and $S\left(h_{i}\right)=g_{i}$, for $i=1, \ldots, k$.

We take a disjoint sequence $\left(C_{n}\right)$ of measurable sets and sequences $\left(x_{i, n}\right)_{n=1}^{\infty}$ in $X$ and $\left(x_{j, n}^{*}\right)_{n=1}^{\infty}$ in $X^{*}$ so that, for $i=1, \ldots, k$ and $j=1, \ldots, l$,

$$
g_{i}=\sum_{n=1}^{\infty} \chi_{C_{n}} \otimes x_{i, n} \quad \text { and } \quad f_{j}=\sum_{n=1}^{\infty} \chi_{C_{n}} \otimes x_{j, n}^{*} .
$$

We define a projection $P$ on $L_{\infty}(\mu, X)$ by

$$
P(f)=\sum_{n=1}^{\infty} \mu\left(C_{n}\right)^{-1} \chi_{C_{n}} \otimes \int_{C_{n}} f d \mu .
$$

Since $\|P(f)\|=\sup _{n}\left\|\mu\left(C_{n}\right)^{-1} \chi_{C_{n}} \otimes \int_{C_{n}} f d \mu\right\|=\sup _{n}\left\|\mu\left(C_{n}\right)^{-1} \int_{C_{n}} f d \mu\right\| \leq\|f\|_{\infty}$, we get $\|P\|=1$. Moreover $P(S(h))=S(h)$, for every $h \in G$. Let $P^{*}$ be the conjugate projection acting on $L_{\infty}(\mu, X)^{*}$. For each $\phi \in L_{\infty}(\mu, X)^{*}$, we define $\left(P^{*} \phi\right)_{n} \in X^{*}$ by

$$
\left\langle\left(P^{*} \phi\right)_{n}, x\right\rangle=\left\langle\phi, \mu\left(C_{n}\right)^{-1} \chi_{C_{n}} \otimes x\right\rangle .
$$

Then $P^{*}(\phi)=\sum_{n=1}^{\infty} \chi_{C_{n}} \otimes\left(P^{*} \phi\right)_{n}$. Indeed, for every $h \in L_{\infty}(\mu, X)$,

$$
\begin{aligned}
\left\langle P^{*}(\phi), h\right\rangle & =\langle\phi, P(h)\rangle \\
& =\sum_{n=1}^{\infty} \mu\left(C_{n}\right)^{-1}\left\langle\phi, \chi_{C_{n}} \otimes \int_{C_{n}} h d \mu\right\rangle \\
& =\left\langle\sum_{n=1}^{\infty} \chi_{C_{n}} \otimes\left(P^{*} \phi\right)_{n}, h\right\rangle .
\end{aligned}
$$

Consequently, $R\left(P^{*}\right) \subset L_{1}\left(\mu, X^{*}\right)$. Moreover, $P^{*}\left(f_{j}\right)=f_{j}$, for every $j=1, \ldots, l$.

We define $L$ as the restriction of $P^{*}$ to the subspace $F$. Let $\phi \in F$ and $h \in G$. Since $\|L\| \leq 1$ and $\langle L \phi, S(h)\rangle=\langle\phi, P(S(h))\rangle=\langle\phi, S(h)\rangle$,

$$
|\langle L \phi, h\rangle-\langle\phi, h\rangle| \leq|\langle L \phi,(I-S) h\rangle|+|\langle\phi,(I-S) h\rangle| \leq \varepsilon\|\phi\|\|h\|,
$$


which is (a) in Theorem 2 Moreover, formulas (11) and (2) imply that, for every $\phi \in F$,

$$
\|L \phi\| \geq\left\|\left.L(\phi)\right|_{G}\right\| \geq\left\|\left.\phi\right|_{G}\right\|-\varepsilon\|\phi\| \geq(1+\varepsilon)^{-1}\|\phi\|-\varepsilon\|\phi\| \geq(1-2 \varepsilon)\|\phi\| .
$$

Hence $L$ is a $2 \varepsilon$-isometry. Moreover, since $\|I-T\|<\varepsilon / 2$ and $L(T(\phi))=T(\phi)$, for every $\phi \in F \cap L_{1}\left(\mu, X^{*}\right)$,

$$
\|L(\phi)-\phi\| \leq\|L(\phi-T(\phi))\|+\|T(\phi)-\phi\|<\varepsilon\|\phi\| .
$$

Thus, an application of Theorem 2 finishes the proof of this part.

(b) The proof is similar. In this case $F$ and $G$ are finite dimensional subspaces of $L_{1}(\mu, X)^{*}$ and $L_{1}(\mu, X)$, respectively. The projection $P$ on $L_{1}(\mu, X)$, defined by

$$
P(f)=\sum_{n=1}^{\infty} \mu\left(C_{n}\right)^{-1} \chi_{C_{n}} \otimes \int_{C_{n}} f d \mu
$$

satisfies $\|P(f)\| \leq \sum_{n=1}^{\infty}\left\|\int_{C_{n}} f d \mu\right\| \leq\|f\|_{1}$, hence $\|P\|=1$. The remainder of the proof is similar to that of part (a).

Remark 5. We have $L_{\infty}\left(\mu, X^{*}\right)=L_{1}(\mu, X)^{*}$ if and only if every $T \in \mathcal{B}\left(L_{1}(\mu), X^{*}\right)$ is representable; equivalently, $X^{*}$ has the Radon-Nikodym property [4].

It was proved in [6] that $L_{\infty}(\mu) \hat{\otimes}_{\epsilon} X^{*}$ is a local dual of $L_{1}(\mu, X) \equiv L_{1}(\mu) \hat{\otimes}_{\pi} X$. In this case, $L_{\infty}(\mu) \hat{\otimes}_{\epsilon} X^{*}=L_{1}(\mu, X)^{*}$ if and only if every $T \in \mathcal{B}\left(L_{1}(\mu), X^{*}\right)$ is compact; equivalently, $X$ is finite dimensional.

Let $K$ denote a compact space. It was proved in [6] that $C(K)^{*} \hat{\otimes}_{\pi} X^{*}$ is a local dual of $C(K, X) \equiv C(K) \hat{\otimes}_{\epsilon} X$. Note that $C(K)^{*} \hat{\otimes}_{\pi} X^{*}=C(K, X)^{*}$ if and only if $X^{*}$ has the Radon-Nikodym property [4].

The following result is a direct consequence of Theorem 4 and Proposition 3 ,

Corollary 6. (a) $L_{\infty}(\mu, X)$ is a local dual of $L_{1}\left(\mu, X^{*}\right)$.

(b) $L_{1}(\mu, X)$ is a local dual of $L_{\infty}\left(\mu, X^{*}\right)$.

Remark 7. Corollary [ 6 is a generalization of the principle of local reflexivity.

\section{REFERENCES}

[1] C. Castaing and A. Valadier. Convex analysis and measurable multifunctions. Lecture Notes in Math. 580. Springer-Verlag, Berlin, 1977. MR 57:7169

[2] P. Cembranos and J. Mendoza. Banach spaces of vector-valued functions. Lecture Notes in Math. 1676. Springer-Verlag, Berlin, 1997. MR 99f:46049

[3] S. Díaz. A local approach to functionals on $L^{\infty}(\mu, X)$, Proc. Amer. Math. Soc. 128 (2000), 101-109. MR 2000c:46066

[4] J. Diestel and J.J. Uhl, Jr. Vector measures. Math. Surveys 15. Amer. Math. Soc., Providence, 1977. MR 56:12216

[5] M. González and A. Martínez-Abejón. Local reflexivity of dual Banach spaces, Pacific J. Math. 189 (1999), 263-278. MR 2000g:46010

[6] M. González and A. Martínez-Abejón. Local dual spaces of a Banach space, Studia Math. 147 (2001), 155-168.

[7] S. Heinrich. Ultraproducts in Banach space theory, J. Reine Angew. Math. 313 (1980), 72104. MR 82b:46013

Departamento de Matemáticas, Facultad de Ciencias, Universidad de Cantabria, E39071 Santander, Spain

E-mail address: gonzalem@unican.es

Departamento de Matemáticas, Facultad de Ciencias, Universidad de Oviedo, E-33007 OVIEDO, SPAIN

E-mail address: ama@pinon.ccu.uniovi.es 